\title{
Odour-taste interactions: A way to enhance saltiness in low-salt content solutions
}

\author{
G. Lawrence ${ }^{\mathrm{a}}$, C. Salles ${ }^{\mathrm{a}}$, C. Septier ${ }^{\mathrm{a}}$, J. Busch $^{\mathrm{b}}$, T. Thomas-Danguin ${ }^{\mathrm{a}, *}$ \\ a INRA, UMR 1129 FLAVIC, Institut National de la Recherche Agronomique, 17 Rue de Sully, F-21000 Dijon, France \\ ${ }^{\mathrm{b}}$ Unilever Food and Health Research Institute, Unilever R\&D Vlaardingen, Olivier van Noortlaan 120, P.O. Box 114, 3130 AC Vlaardingen, The Netherlands
}

\section{A R T I C L E I N F O}

\section{Article history:}

Received 10 June 2008

Received in revised form 8 October 2008

Accepted 9 October 2008

Available online 17 October 2008

\section{Keywords:}

Saltiness

Enhancement

Odour-induced taste perception

\section{Introduction}

Food flavour is an important cue in daily life since it constitutes a main driver of food appreciation, acceptance and choice. The overall perception of flavour is considered as an integration of simultaneous sensory perceptions including taste and odour. It has been demonstrated that taste can increase odour intensity and conversely, the perception of taste can be enhanced by odour (Salles, 2006). The most studied intermodal odour-taste interaction is between sweetness and odour. It has been established that a condition to observe such perceptual interactions is the congruency between the considered taste and odour. Frank and Byram (1988) observed an increase of sweetness by adding strawberry odour to beaten cream but they did not observe this phenomenon with peanut butter. Strawberry odour was not able to enhance saltiness. Stevenson, Prescott, and Boakes (1999), studied the effect of several odour notes on sweetness and sourness. They found that some food-like odours (caramel, maracudja, lychee) enhanced sweetness and some non-food-like odours (maltol, damascone, angelique) suppressed sweetness. For a given odour, this effect may be dependant upon the aroma concentration (Cliff \& Noble, 1990; Hewson, Hollowood, Chandra, \& Hort, 2008; Schifferstein \& Verlegh, 1996; Stevenson et al., 1999). Other studies reported odour-taste interactions with sourness or bitterness (Salles, 2006) and only Djordjevic, Zatorre, and Jones-Gotman (2004b) reported an enhancement of saltiness by soy sauce odour.

\footnotetext{
* Corresponding author. Fax: +33 0380693227

E-mail address: Thierry.Thomas-Danguin@dijon.inra.fr (T. Thomas-Danguin).
}

Several studies were performed in order to find neurophysiological correlates of odour-taste integration (Verhagen \& Engelen, 2006). Single-cell recording studies in monkeys indicated that integration across sensory modalities is reflected in the presence of multimodal neurons, in the orbitofrontal cortex, that receive converging sensory information (Rolls \& Baylis, 1994). Neuroimaging studies confirmed these findings and showed differences in neuronal activation patterns, especially in the orbitofrontal cortex, between unimodal stimulation with a taste or an odour and bimodal (simultaneous) presentation of the same tastes and odours (Small \& Prescott, 2005). These neurophysiological studies clearly indicate that odour-taste integration does occur at the neural level. The cognitive origin of such effects is now generally admitted (Prescott, 2004; Valentin, Chrea, \& Nguyen, 2006). Indeed, it has been reported that imagined odours influence taste perception in the same way as perceived odours. Djordjevic, Zatorre, and Jones-Gotman (2004a) showed that the accuracy of detecting low sucrose concentrations was better when a strawberry odour was imagined during the detection task as compared to ham odour. This pattern was found to parallel the one observed when the actual odours were presented, although to a smaller extent.

In recent years, health concerns related to salt have led to extensive research on low-salt foods. Indeed, international authorities, such as the World Health Organisation (WHO), advocate sodium reduction in foods. The WHO currently recommends a salt intake of $5 \mathrm{~g}$ per day (World Health Organisation, 2007). Decreasing the sodium content in food products without changing consumer acceptability has become an important challenge for the 
food industry. One of the main consequences of decreasing salt content is that the sensory characteristics are affected. Sodium chloride is generally present in significant quantities in products like bread, soup, cheese and sausages. In addition to taste enhancing properties, sodium chloride has also a role in, e.g. texture, aroma release, preservation of food against microorganisms and as a fermentation aid. Some studies showed that a significant decrease of sodium chloride content in some food products did not affect consumer appreciation (Girgis et al., 2003). However, it cannot be generalized because a decrease in sodium content is often associated with a decrease in consumer acceptance (Breslin \& Beauchamp, 1997).

Only a few studies reported the enhancement of saltiness by odours. Such an effect could be of interest for product developers as one can hypothesize to use aromas to compensate salt reduction in food. In the present study, our aim was to test this hypothesis. We especially investigated whether well selected odours can enhance saltiness of low-salt content solutions. As a sensory selection of odours associated with saltiness would be time consuming, we took advantage of the odour-taste cognitive association and screened a large number of odours on the basis of their semantic attribute only. Thus, in the first experiment we selected odours that evoked saltiness on the basis of their name. In the second experiment we recorded saltiness of solutions containing the selected odours, with and without sodium chloride, in order to validate the effectiveness of odour-induced changes in saltiness.

In this paper, the term "aroma" refers to the volatile compound (e.g. the commercially available aromatic compositions). The term "odour" refers to the percept which results from the perception of the volatiles whereas the term "flavour" refers to the perceptual combination of odour and taste.

\section{Materials and methods}

\subsection{Experiment 1}

\subsubsection{Subjects}

Eighty-one French panellists (aged 19-73 years, 56 women) received 10 euros for their participation in a 1-h session. They did not receive any information about the aim of the experiment.

\subsubsection{Procedure}

Eighty-six food names were used in this experiment. Most of them were pre-selected based on their association with salty food and a few control names which were not associated with salty food, such as lemon (for sourness), vanilla, strawberry or milk chocolate (for sweetness). For each food name, panellists were asked to estimate taste intensity (bitterness, sourness, saltiness, and sweetness) on four linear scales from 0 to 10 ( 0 : none and 10: extremely strong) or to indicate "not known" if the food name was unknown to them. In this session, the order of presentation of the food names was randomized for each subject. Data acquisition was performed with FIZZ software (Biosystèmes, Couternon, France). Panellists were placed in separate booths, at room temperature $\left(21^{\circ} \mathrm{C}\right)$.

\subsection{Experiment 2}

\subsubsection{Subjects}

Fifty-nine French panellists (aged 21-67 years, 42 women) were split into two groups included 29 and 30 panellists, respectively. Each panellist participated in two 1-h sessions and received 20 euros for their participation. They were not informed of the aim of the experiment. All participants reported normal ability to perceive smell and taste. They were requested not to smoke or eat $1 \mathrm{~h}$ before the session.

\subsubsection{Stimuli}

Twenty commercial aromas were selected to fit with 20 of the food names used in experiment 1 . Nineteen aromas had been rated as salty and a control (carrot) had not. Triangular tests with noseclip (aromas without salt in water vs. water) were performed in order to check that aromas solutions were tasteless. Five of the tested aromas were found to be tasty and they were removed from data analysis. The aromas, their supplier and used concentrations are presented in Table 1. The aroma concentrations were chosen according to their intensity and acceptability in a preliminary test (data not shown).

In the orthonasal condition, aromas were presented in mineral water (Evian, France). In the retronasal condition, aromas were either presented in Evian mineral water ("without salt" condition) or in Evian mineral water containing food grade sodium chloride (Jerafrance, Jeufosse, France) at a weak concentration of $0.02 \mathrm{M}$ ("with salt" condition).

All solutions were made $24 \mathrm{~h}$ prior testing and they were stored in glass bottles at $4{ }^{\circ} \mathrm{C}$ until use.

\subsubsection{Procedure}

In this experiment, the first group of 30 consumers tested 10 flavours following a complete block design without replicates. The second group tested 10 other flavours following the same experimental design. Each panellist participated in two 1-h sessions within the same week. During a session, five flavours were evaluated in two sensory conditions. At first, panellist rated the stimulus orthonasally (orthonasal condition) and then in mouth (retronasal condition).

For each sample, panellists were asked to rate odour intensity and taste intensity (sourness, bitterness, saltiness, and sweetness) on five linear scales from 0 to 10 . We asked them to rate odour intensity in order to include this intensity in the data analysis as the aroma concentrations were not adjusted for iso-intensity. Moreover, rating odour intensity in addition to the four tastes should avoid dumping. The dumping effect is a bias due to omission of potentially salient raring scales (Clark \& Lawless, 1994). Thus, when subjects are unable to rate sensory qualities that may be present in a sample (e.g. odour in a flavour sample), they 'dump' these qualities onto ratings of other qualities that are rated (e.g. saltiness), thereby producing apparent enhancement. Using multiple and appropriate scales prevent this bias to occur (Nguyen, Valentin, Ly, Chrea, \& Sauvageot, 2002; Prescott, Johnstone, \& Francis, 2004; Valentin et al., 2006).

Panellists were placed in separate booths, at room temperature $\left(21^{\circ} \mathrm{C}\right)$. Samples were presented under red light in a randomized order, different for each panellist.

\section{Table 1}

Aromas, suppliers and concentrations used in experiment 2.

\begin{tabular}{lll}
\hline Name & Supplier name & Concentration used in solution $(\mathrm{g} / \mathrm{l})$ \\
\hline Anchovy & Symrise & 0.25 \\
Bacon & Givaudan & 0.25 \\
Carrot & Givaudan & 0.1 \\
Chicken & Givaudan & 1 \\
Comté cheese & Symrise & 0.02 \\
Concentrated cheese & Symrise & 0.001 \\
Goat cheese & Symrise & 0.05 \\
Ham & Givaudan & 0.7 \\
Peanuts & Givaudan & 0.75 \\
Roquefort cheese & Symrise & 0.25 \\
Sardine & Givaudan & 0.5 \\
Soy sauce & Symrise & 0.6 \\
Sotolon & Sigma-Aldrich & 0.006 \\
Tomato & Givaudan & 0.8 \\
Tuna & Givaudan & 0.5 \\
\hline
\end{tabular}

a Sotolon: 4,5-dimethyl-3-hydroxy-2,5-dihydrofuran-2-one. 


\subsubsection{Orthonasal condition}

Sixty milliliter opaque glass bottles (VWR International, France) were filled with $9 \mathrm{ml}$ of aroma solutions. Panellists were asked to smell the odour and to evaluate it. Between each sample, a 30 s rest was imposed.

Orthonasal evaluation was performed with all solutions without salt.

\subsubsection{Retronasal condition}

During one session, 12 samples were evaluated: five of the aroma solutions were offered in the "with" and the "without salt condition" in addition to two blanks (Evian water and Evian water with $0.02 \mathrm{M}$ sodium chloride). Twenty milliliter of each sample was presented in $80 \mathrm{ml}$ plastic cup. Panellists were requested to pinch their nostrils, to sip the solution and to unblock their nostrils before rating the solution to avoid any orthonasal perception before introduction of the sample in mouth. The interval between each sample was $90 \mathrm{~s}$. Panellists were asked to cleanse their mouth with bread (without salt) and Evian water.

\subsection{Data analyses}

Data analyses were carried out with STATGRAPHICS CENTURION XV.I Software (Version 15.2, Sigma-plus, France).

For experiment 1, Principal Component Analysis (PCA) was carried out with food names as variables. Analysis of variance (ANOVA) was performed using a GLM (General Linear Model) procedure where panellists were considered as random factors. Post-hoc comparison of LS (Least Square) means was performed with the Student Newman Keuls test.

For experiment 2, a GLM procedure for ANOVA and analysis of co-variance (ANCOVA), where panellists were considered as random factors, was performed. The adjustment for multiple comparisons between the saltiness intensity of the sample and the reference (water or water with salt) was performed with the Dun- nett test. To analyze correlations between variables, Pearson's correlation coefficients were calculated.

For all data analysis, effects were considered to be significant when $p<0.05$.

\section{Results}

\subsection{Experiment 1: Name condition}

In this experiment 86 food names were judged on their taste associations. A PCA was performed in order to compare the different tastes associated with the food names (Fig. 1). The two first principal components accounted for $79 \%$ of the total variability. The first principal component accounted for $56 \%$ of the total variance and separated saltiness from other tastes. The second component accounted for $23 \%$ of the total variance and separated saltiness and sweetness from bitterness and sourness.

For saltiness, a two-ways ANOVA (judge, food names) revealed a significant effect of judge $(F(80,6387)=31.9, p<0.0001)$ and food names $(F(85,6387)=81.1, p<0.0001)$. Post-hoc analysis indicated that anchovy, bacon, smoked salmon, dry sausage, peanuts, "bouillon cube" and sardine were evaluated as the most salty whereas vanilla, orange, fig, strawberry, milk chocolate, cinnamon and lemon were evaluated as the least salty (Fig. 2).

It is noteworthy that a significant Pearson's correlation $(r=0.47 ; p<0.0001)$ exists between the mean associated saltiness of the food names and the reported sodium content of these food products (Favier, Ireland-Ripert, Toque, \& Feinberg, 1995). This correlation was performed on 79 of the food names used in the present study (vanilla, four spices, nutmeg, shallot, cucumber, clove and cinnamon were not included in this correlation).

\subsection{Experiment 2: Orthonasal and retronasal conditions}

In this experiment 20 water solutions of commercial aromas with or without sodium chloride added - were rated on several

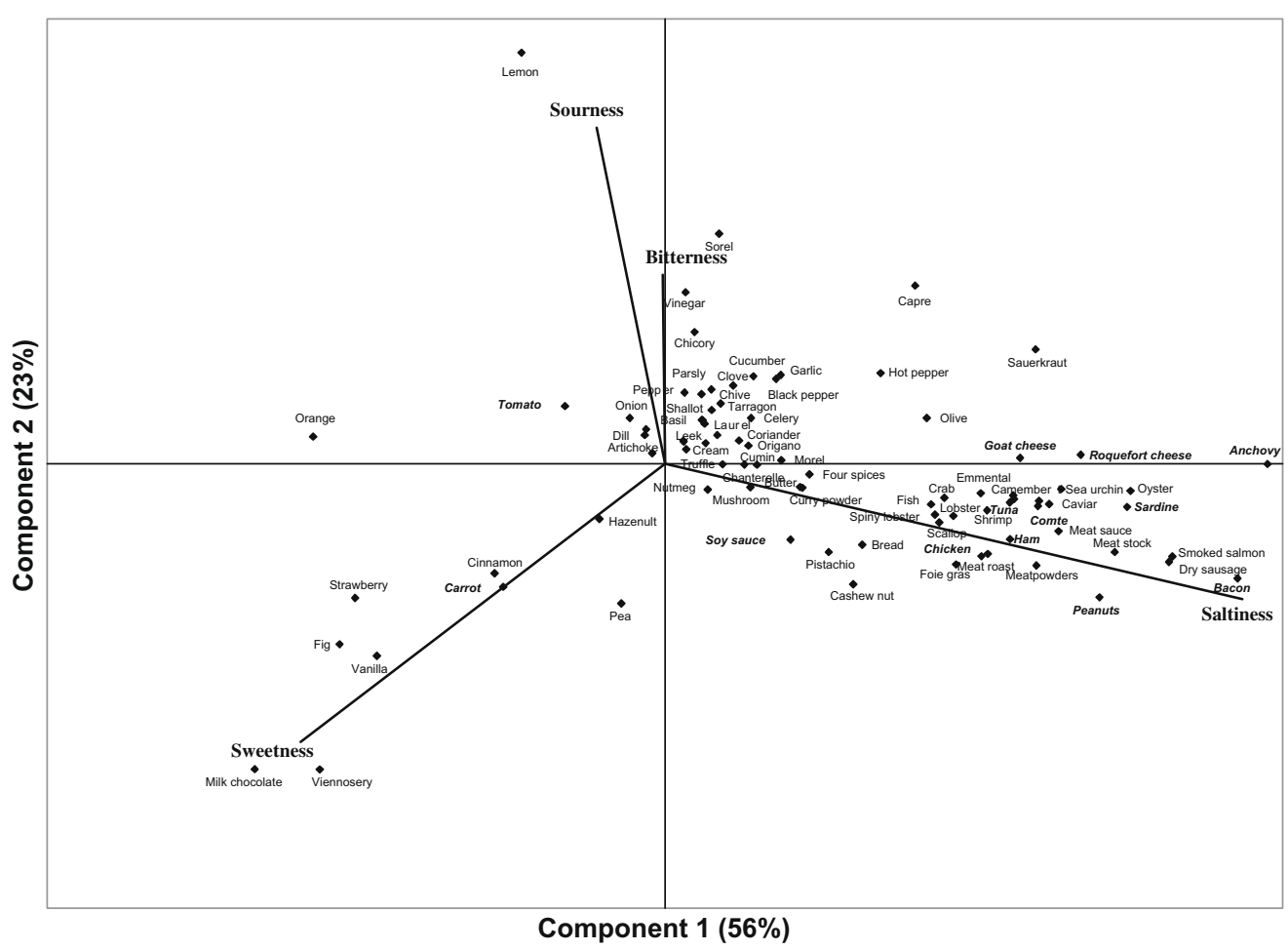

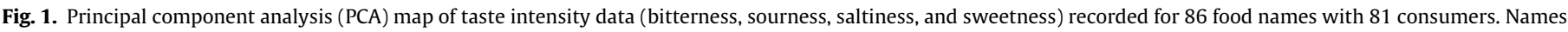
in italic, represents aromas kept for experiment 2. 


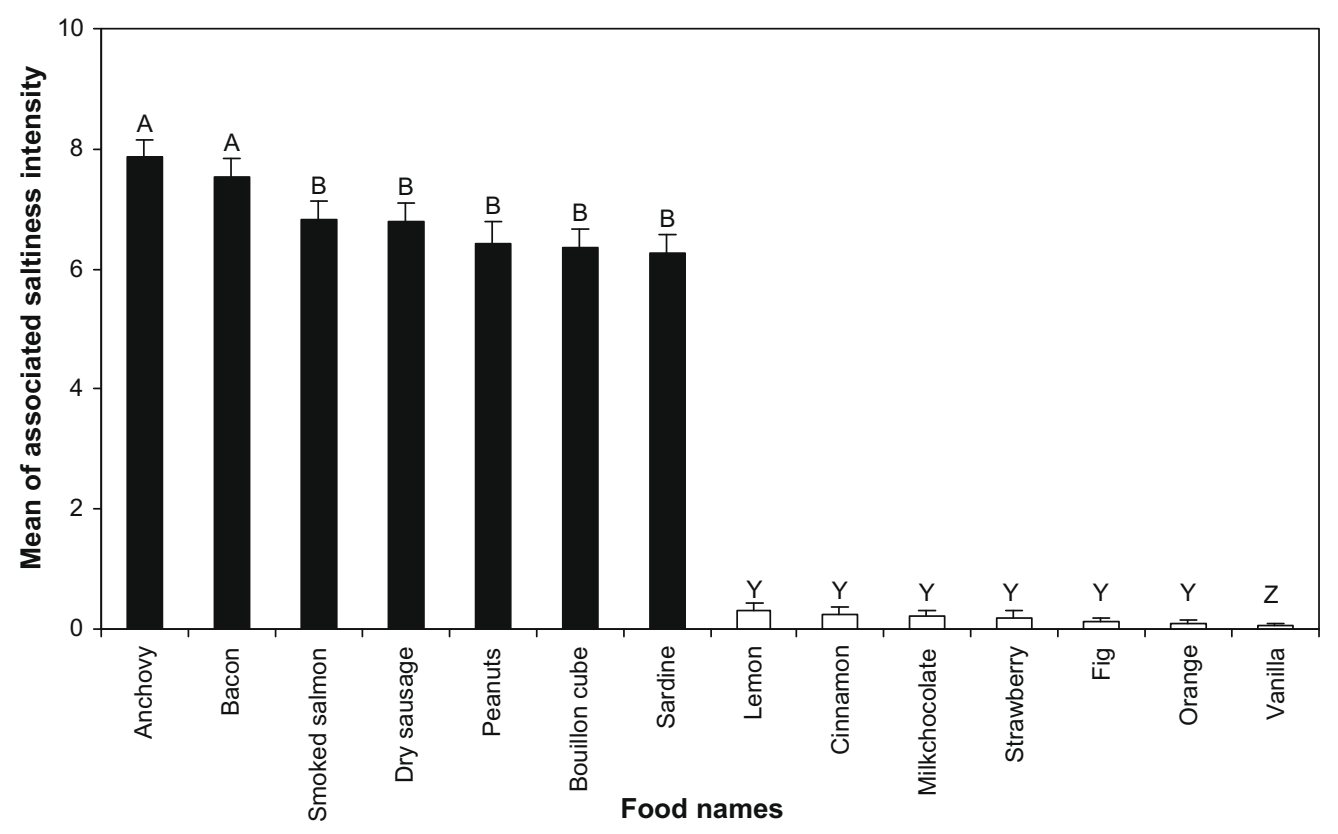

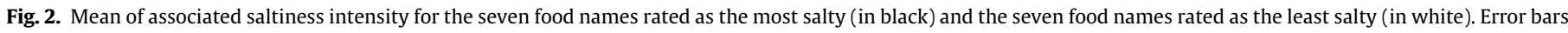
represent the standard error of the mean. The same letters indicate that saltiness intensities are not significantly different at a level of $5 \%$.

sensory attributes either orthonasally (smelling the solution) or retronasally (solution in the mouth). Only 15 aroma solutions and a reference (water) were kept for data analysis because five aroma solutions (without salt added) were found to be tasty (triangular tests, data not shown).

\subsubsection{Orthonasal}

A two-ways ANCOVA (judge, aroma solution, odour intensity as covariate) on saltiness, sweetness, sourness and bitterness was performed.

For saltiness $(M=3.1, \mathrm{SD}=3.2)$, the ANCOVA revealed a significant effect of judge $(F(58,485)=2.5 ; p<0.0001)$, aroma solution $(F(15,485)=8.9 ; p<0.0001)$ and odour intensity $(F(1,485)=25.3$; $p<0.0001)$. Post-hoc analyses showed that 10 of the 15 odours were rated saltier than water (reference; Fig. 3a). For sweetness $(M=1.3, \mathrm{SD}=2.2)$, significant effects of judge $(F(58,485)=1.5$; $p=0.01)$, aroma solution $(F(15,485)=6.7 ; p<0.0001)$ and odour intensity $(F(1,485)=7.6 ; p=0.006)$ were obtained. Post-hoc analyses revealed that tomato odour only was significantly sweeter than the reference. For sourness $(M=1.4, \mathrm{SD}=2.1)$, significant effects of judge $\quad(F(58,485)=3.0 ; \quad p<0.0001)$, aroma solution $(F(15,485)=5.7 ; p<0.0001)$ and odour intensity $(F(1,485)=11$; $p=0.001$ ) were obtained. Post-hoc analyses revealed that anchovy, carrot, comté cheese and roquefort cheese odours were rated with a higher sourness than the reference. For bitterness $(M=1.9$, $\mathrm{SD}=2.6)$, significant effects of judge $(F(58,485)=2.5 ; p<0.0001)$, aroma solution $(F(15,485)=3.0 ; p=0.0001)$ and odour intensity $(F(1,485)=11.1 ; p=0.0009)$ were obtained. Post-hoc analyses revealed that anchovy, ham, bacon, chicken and roquefort cheese odours were significantly more bitter than the reference.

\subsubsection{Retronasal - without salt}

In the retronasal and "without salt" condition (Fig. 3b), a twoways ANCOVA (judge, aroma solution, odour intensity as covariate) on saltiness ratings $(M=2.3, \mathrm{SD}=2.7)$, indicated a significant effect of judge $(F(58,485)=2.9 ; p<0.0001)$, aroma solution $(F(15,485)=3.3 ; \quad p<0.0001)$ and odour intensity $(F(1,485)=46.2 ; p<0.0001)$. Post-hoc analyses showed a significant difference in saltiness ratings for two flavours (sardine and ham) as compared to water (reference) and only a trend for the comté cheese flavour.

It is noteworthy that without correction for odour intensity, 10 flavours (sardine, ham, bacon, anchovy, peanuts, chicken, tuna, comté cheese, and roquefort cheese) were rated as saltier than water.

A two-ways ANCOVA (judge, aroma solution, odour intensity as covariate) on sweetness ratings $(M=0.7, \mathrm{SD}=1.6)$, indicated significant effects of judge $(F(58,485)=2.9 ; p<0.0001)$, aroma solution $(F(15,485)=6.0 ; p<0.0001)$. Odour intensity was not found to be a significant factor $(p>0.9)$. Post-hoc analyses revealed that peanut, carrot and tomato flavours were significantly sweeter than the reference (water). For sourness $(M=1.2, \mathrm{SD}=1.9)$, significant effects judge $(F(58,485)=3.2 ; \quad p<0.0001)$, aroma solution $(F(15,485)=2.5 ; p=0.002)$ and odour intensity $(F(1,485)=13.9$; $p=0.0002$ ) were obtained. Post-hoc analyses revealed that roquefort cheese flavour only was rated with a higher sourness than the reference. For bitterness $(M=2.3, \mathrm{SD}=2.8)$, significant effects of judge $\quad(F(58,485)=3.0 ; \quad p<0.0001), \quad$ aroma solution $(F(15,485)=3.5 ; p<0.0001)$ and odour intensity $(F(1,485)=25.2$; $p<0.0001)$ were obtained. Post-hoc analyses revealed that anchovy, ham, bacon and roquefort flavours were significantly more bitter than the reference.

\subsubsection{Retronasal - with salt}

Concerning the retronasal and "with salt" condition (Fig. 4a), a two-ways ANCOVA (judge, aroma solution, odour intensity as covariate), on saltiness ratings $(M=3.4, \mathrm{SD}=3.0)$ indicated a significant effect of judge $(F(58,485)=2.9 ; p<0.0001))$, aroma solution $(F(15,485)=2.5 ; p=0.002)$ and odour intensity $(F(1,485)=53$; $p<0.0001)$. Post-hoc analyses showed that only one flavour, carrot, was significantly different. A decrease of the perceived saltiness was observed when this aroma was included in the $\mathrm{NaCl}$ solution.

A two-ways ANCOVA (judge, aroma solution, odour intensity as covariate) for sweetness $(M=0.7, \mathrm{SD}=1.5)$, indicated significant effects of judge $(F(58,485)=2.7 ; p<0.0001)$, aroma solution $(F(15,485)=7.0 ; p<0.0001)$. Odour intensity was not found to be a significant factor $(p>0.2)$. Post-hoc analyses revealed that peanut, carrot and tomato flavours were significantly sweeter than 


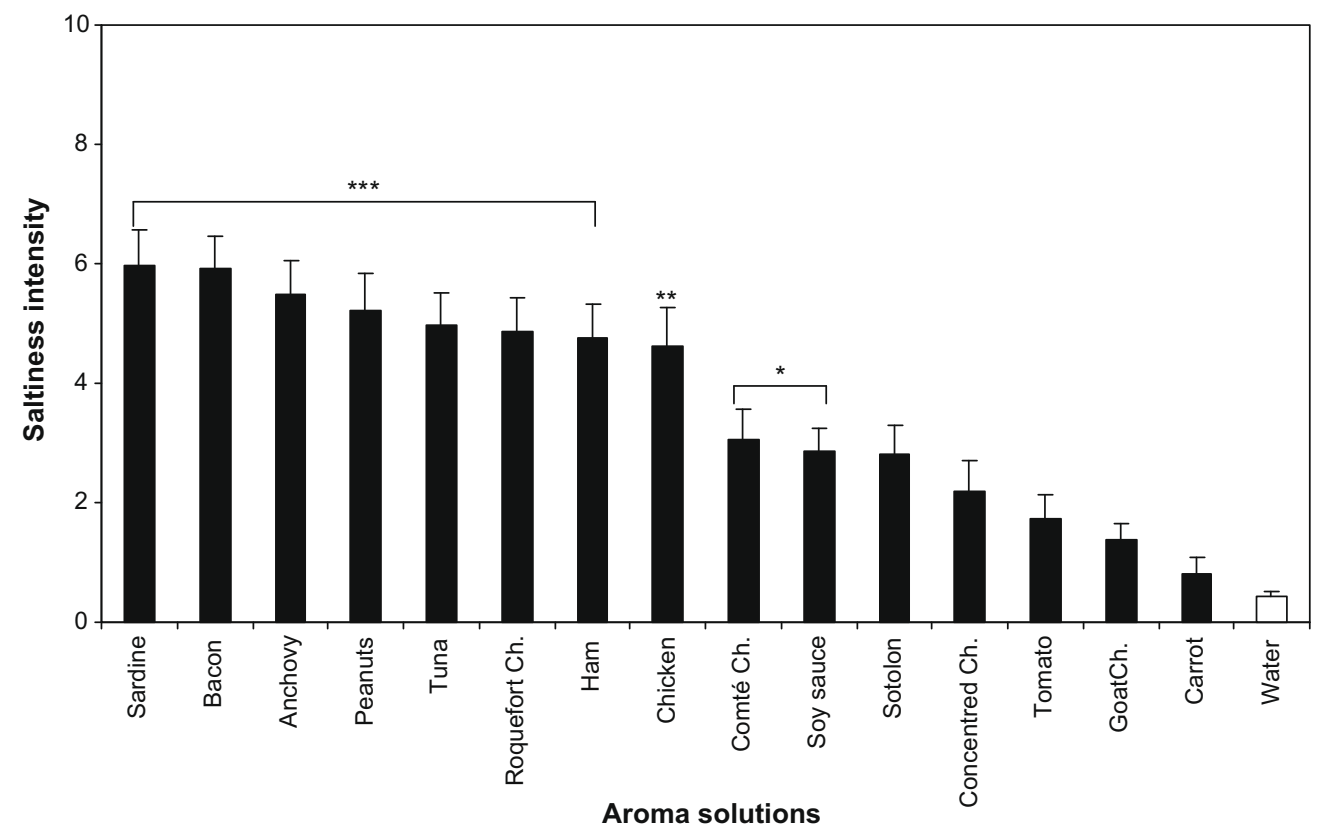

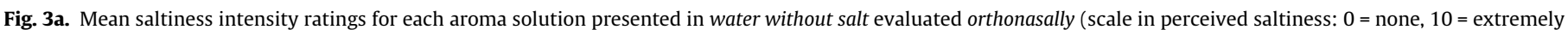

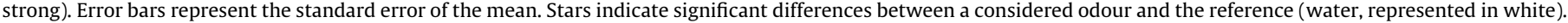
${ }^{* * *} p<0.001 ;{ }^{* *} p<0.01 ;{ }^{*} p<0.05$. Ch. $=$ Cheese.

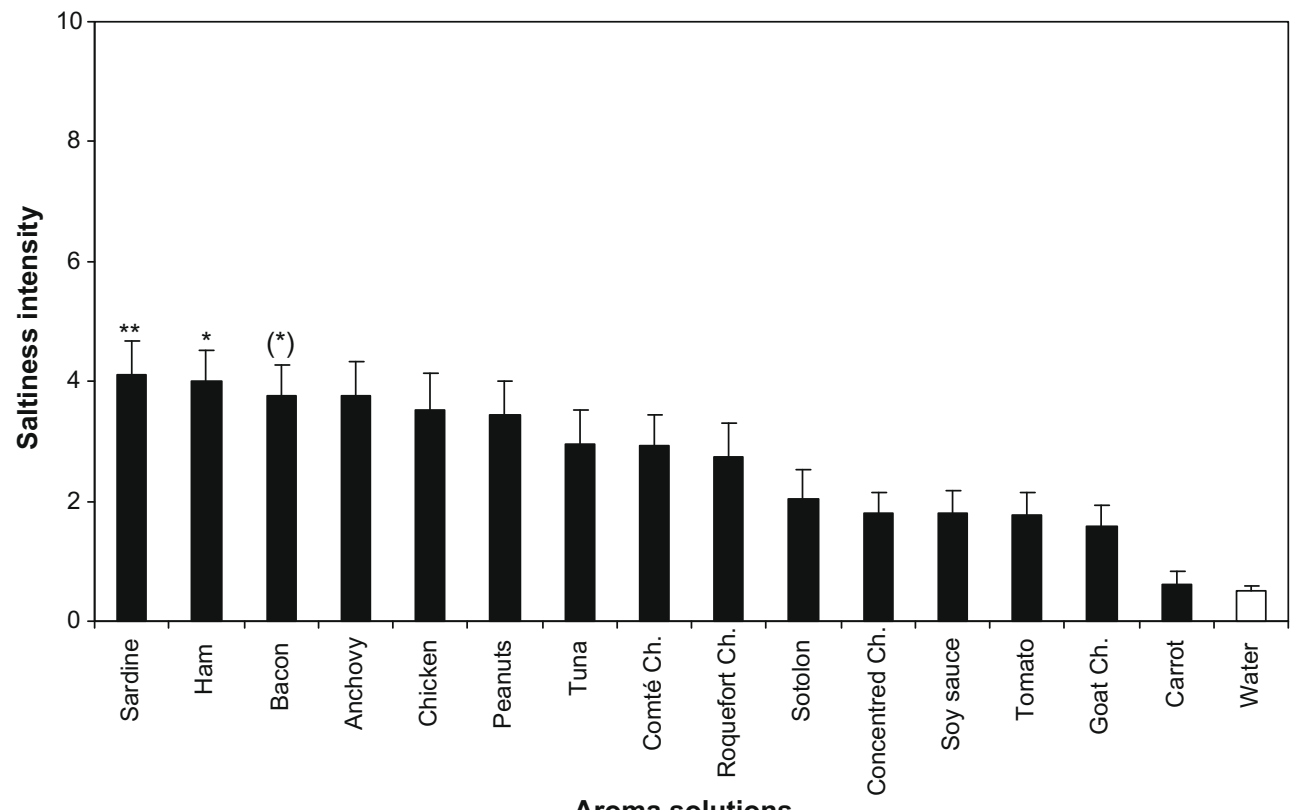

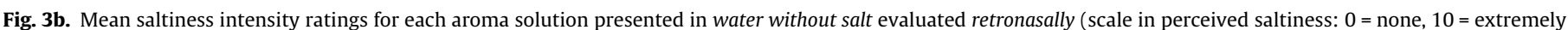

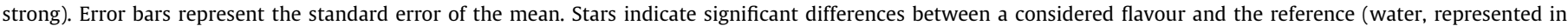
white) taking in account the odour intensity in an ANCOVA. ${ }^{* *} p<0.01 ;{ }^{*} p<0.05 ;\left({ }^{*}\right) p<0.1$. Ch. $=$ Cheese.

the reference (water). For sourness $(M=1.1, \mathrm{SD}=1.8)$, significant effects of judge $(F(58,485)=2.7 ; p<0.0001)$, aroma solution $(F(15,485)=2.4 ; p=0.003)$ and odour intensity $(F(1,485)=6.9$; $p=0.009$ ) were obtained. Post-hoc analyses revealed that bacon and roquefort cheese flavours were rated with a higher sourness than the reference (water). And, for bitterness $(M=2.3, \mathrm{SD}=2.9$ ), significant effects of judge $(F(58,485)=3.9 ; p<0.0001)$, aroma solution $(F(15,485)=5.6 ; \quad p<0.0001)$ and odour intensity $(F(1,485)=12.5 ; p=0.0005)$ were obtained. Post-hoc analyses revealed that anchovy, comté cheese, concentrated cheese, ham, ba- con chicken and roquefort cheese flavours were significantly more bitter than the reference (water).

In order to evaluate the increase of perceived saltiness when adding an aroma to the salt solution, we performed a two-ways ANOVA (judge, aroma solution) on saltiness ratings. The results indicated a significant effect of judge $(F(58,486)=3 ; p<0.0001)$ and aroma solution $(F(15,486)=7.5 ; p<0.0001)$. Post-hoc analyses revealed that nine commercial aroma solutions were saltier than the reference (water + salt). In Fig. $4 \mathrm{~b}$, we represent the "odour induced saltiness enhancement" (OISE), namely the difference be- 


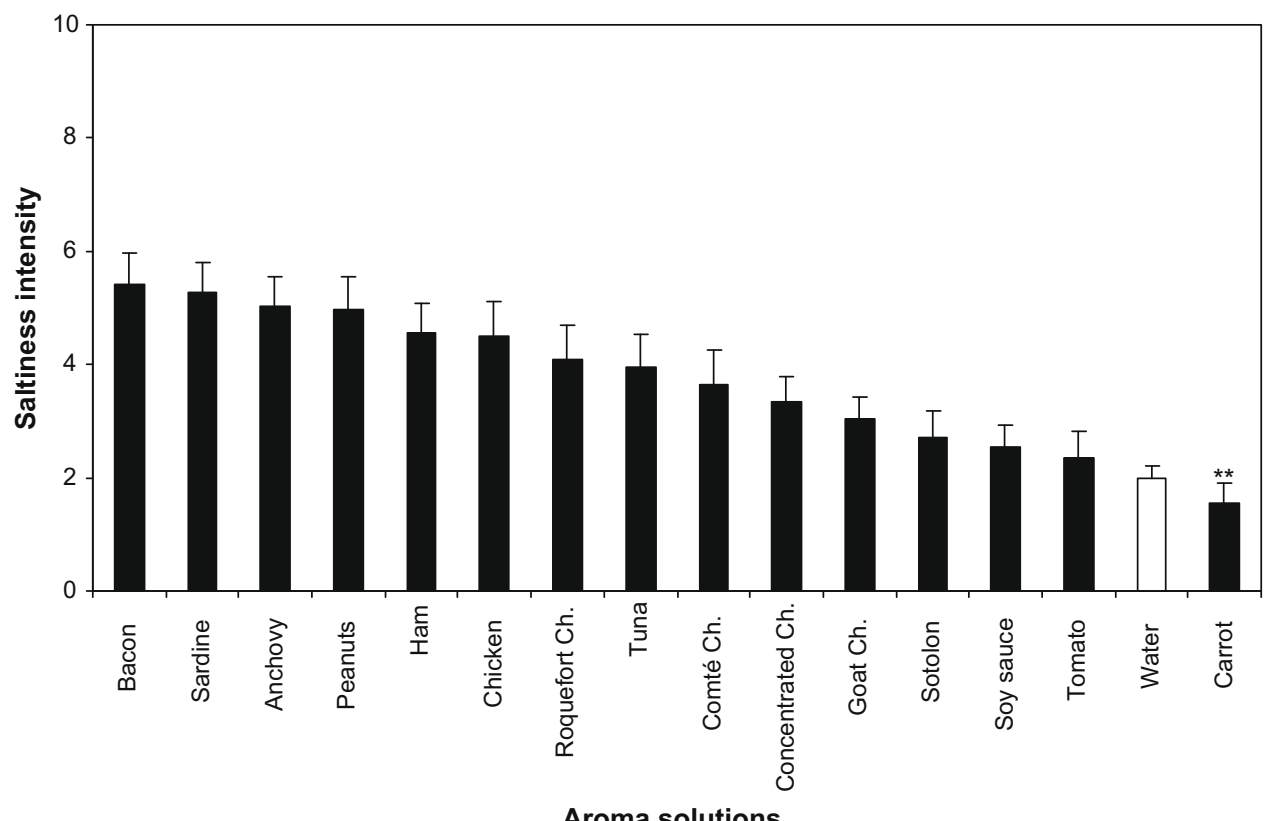

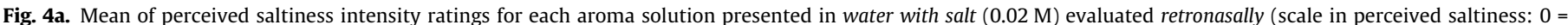

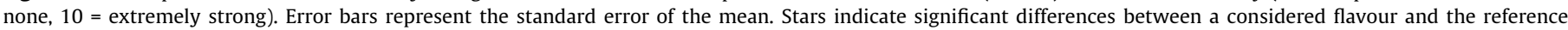
(water + salt, represented in white). ${ }^{* *} p<0.01$. Ch.= Cheese.

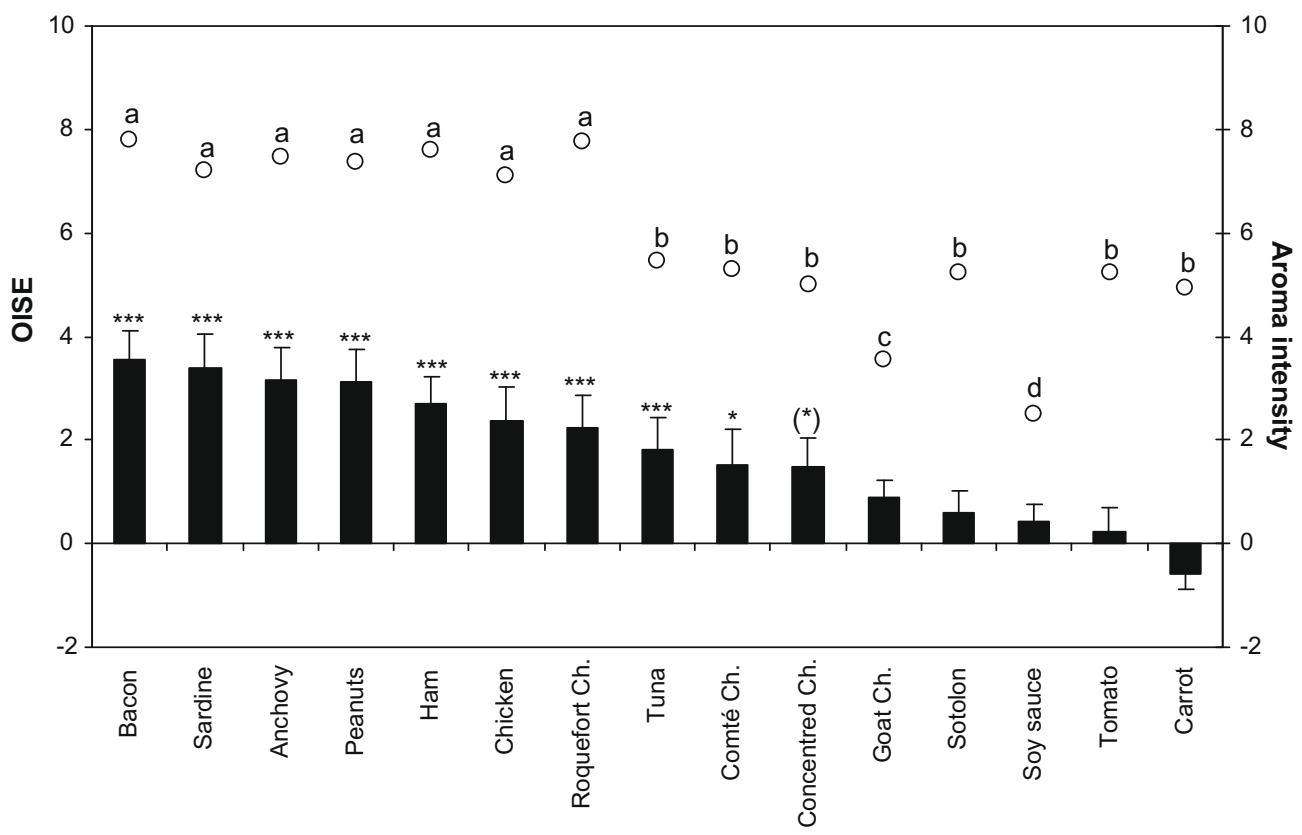

Odorant solutions

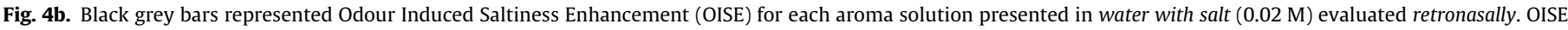

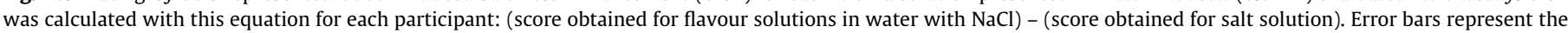

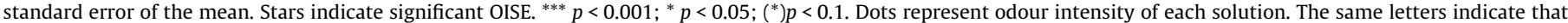
odour intensity is not significantly different at a level of $5 \%$. Ch. = Cheese.

tween (i) the mean saltiness for the solutions containing an aroma and a low concentration of $\mathrm{NaCl}$ and (ii) the mean saltiness for the solution containing only the same amount of $\mathrm{NaCl}$. Means for OISE and post-hoc comparisons are thus reported in Fig. $4 \mathrm{~b}$. In order to show the importance of odour intensity for saltiness perception, we performed a two-ways ANOVA (judge, aroma solution) on odour intensity. The results indicated a significant effect of judge $(F(58,486)=2.5 ; \quad p<0.0001) \quad$ and aroma solutions
$(F(15,486)=45.3 ; p<0.0001)$. Post-hoc analyses revealed differences between flavours which are reported in Fig. 4b.

\section{Discussion}

In this study, our aim is to investigate whether well selected odours can enhance saltiness perception. Our results showed that odours can especially enhance saltiness in simple water solutions 
containing a small amount of salt $(\mathrm{NaCl})$. While odour induced taste enhancement were previously reported for several tastes such as sweetness (Djordjevic et al., 2004b; Frank, Van der Klaauw, \& Schifferstein, 1993; Schifferstein \& Verlegh, 1996; Stevenson et al., 1999), bitterness (Labbe, Damevin, Vaccher, Morgenegg, \& Martin, 2006), sourness (Saint-Eve, Paci Kora, \& Martin, 2004; Stevenson et al., 1999), only few examples have been reported for odour induced saltiness enhancement. Pionnier et al. (2004) suggested that saltiness could be enhanced by a cheese odour. Djordjevic et al. (2004b) found specific taste-smell interactions where strawberry odour enhanced sweetness and soy sauce odour enhanced saltiness. They especially showed that odour induced saltiness enhancement (OISE) occurred when the odour is actually presented (without physical contact between tastant and odorant) or only imagined. They concluded that OISE is a centrally mediated phenomenon relying on taste and smell integration as also suggested by Small and Prescott (2005). In our study, we extended this OISE finding using several odours elicited by solution containing or not sodium chloride. We confirmed that odour-taste integrations are specific of taste and odour association. Our results showed that several salt-associated odours could induce saltiness enhancement. But we also showed that odours not associated with salt could induce saltiness reduction: carrot odour induced a decrease of saltiness ratings in a low-salt content solution.

In our study, we evaluated smell-taste congruency in an original way. In the first experiment, we asked a panel of 81 consumers to rate associated tastes of food products as evoked by verbal items (written food names). The results evidenced significant differences in saltiness between food names. Anchovy and bacon items were especially considered as the most saltiness-associated food names. Our results also demonstrated that such intensity ratings are subjective measures of perception or expectations that are largely influenced by our knowledge and experience (Schifferstein, 1997). Indeed we were able to find a significant correlation between saltiness ratings for the food names and actual salt content of the food. Most interestingly, we found a strong correlation between OISE level and evoked saltiness as rated for food names (Pearson correlation $r(13)=0.90 ; p<0.0001$ ). Anchovy and bacon, both items that evoked the highest saltiness in experiment 1 were also found to be the two odours that elicited the highest OISE in the retronasal with salt condition of experiment 2 . Taking together, these results suggest that rating sensory dimensions of odours only evoked by their names (names of their food source) could be a very quick and easy way of selecting odours that potentially induce changes in taste perception. Such a finding is in line with the results of Djordjevic et al. (2004b) who found that saltiness enhancement (OISE) induced by soy sauce odour occurred when the odour was only imagined. Summarizing, OISE is a centrally mediated phenomenon based on associative memory (Small \& Prescott, 2005; Stevenson \& Tomiczek, 2007; Verhagen \& Engelen, 2006) and only the activation of an internal representation of a salt-associated odour (e.g. induced by odour source) is sufficient to induce the enhancement. Moreover, the saltiness dimension of the internal odour representation is a good predictor of OISE level.

Our data revealed saltiness differences between tasteless aroma solutions evaluated orthonasally or retronasally (Fig. 3a and b). We especially found the saltiness score to be higher when the odour was perceived orthonasally. Once in the mouth, most of the tested aroma solutions were not perceived as significantly more salty than water. This is the same findings as reported by Sakai, Kobayakawa, Gotow, Saito, and Imada (2001). They reported slight differences in sweetness enhancement of aspartame solutions when a vanilla odour was presented, separately from the tastant, either orthonasally or retronasally. In our case, one can argue that the absence of actual salt stimulation on the tongue could have led to a decrease of the saltiness intensity evoked by the odour. Indeed the absence of actual taste system stimulation could have softened the cognitive influence of odour on taste association. This explanation is in line with the concept of perceptual interactions between taste and smell. These are cognitive interactions supported by associative memory but should also rely on an effective multisensory integration: when the taste input is not there, the odour-taste association is less likely to operate. An alternative explanation could be that, upon retronasal presentation, the perceived odour intensity was lower and thus saltiness ratings were also lower (means perceived odour ratings retronasally and orthonasally, respectively 4.5 and $4.9 ; t=2.1, p<0.05$ ). Kuo, Pangborn, and Noble (1993) showed that the retronasal intensity of a citral solution was lower than the orthonasal intensity. They explained that the retronasal intensity could be affected by several factors such as movement and temperature of the mouth, saliva effects and chemical reactions. Linforth, Martin, Carey, Davidson, and Taylor (2002) reported that the headspace of several aroma solutions was higher than the corresponding nosespace. These results supported the idea that the concentration of volatiles reaching the olfactory receptors is higher via the orthonasal route than the retronasal route in the case of aqueous solutions. These authors suggested that saliva was not the main factor affecting volatile delivery in mouth and that the decrease of volatile concentration through the retronasal route was mainly determined by the mass transfer from the solution to the gas phase. Further experiments are needed to test these hypotheses. It is also possible that when tastes are induced by an odour, perceptual interactions between induced tastes (Keast \& Breslin, 2002) take place. In our experiment, such perceptual interactions could have occurred retronasally. Indeed odourinduced bitterness was found to be higher in both retronasal conditions as compared to the orthonasal one. This induced bitterness could thus have counteracted induced saltiness and OISE.

Whatever experimental condition, our statistical analyses revealed a significant effect of odour intensity (covariate) on saltiness: the higher the odour intensity the higher the saltiness. As a consequence, it is likely that the odour intensity is a good predictor of the saltiness increase induced by the odour. This result confirms the findings of Hewson et al. (2008), Stevenson et al. (1999) and Cliff and Noble (1990) for sourness and sweetness.

However, for tomato and carrot odours, even though the odour intensity was quite high, the OISE remained very low or even negative. It can be seen in Fig. 1 that both odour representations were not associated with saltiness. Carrot and tomato aromas used in this experiment were indeed found to evoke sweetness. Perceptual interactions between tastes (Keast \& Breslin, 2002) usually showed sweetness to reduce saltiness. Thus it is possible that, in our experimental conditions, induced sweetness counteracted actual saltiness of $\mathrm{NaCl}$ which could explain why tomato and carrot odours, despite their medium odour intensity, were found not to enhance saltiness. These findings show that for odours to evoke OISE, a cognitive association with saltiness is required. More generally, our results suggest that to induce taste enhancement, an odour should be associated with that specific taste. Secondly, the odour intensity of this odour is the driver of the OISE power. From this perspective, it is interesting to consider goat cheese or soy sauce odour. Indeed, Djordjevic et al. (2004b) found a significant OISE using soy sauce odour. In our data soy sauce did not show a significant OISE. Beyond the likely differences in odour quality of our flavour and the one used by these authors and further possible cultural differences, the intensity of our soy sauce odour is the lowest of our tested odours. This could explain, at least in part why we did not find a significant OISE using soy sauce odour.

To conclude, we presented an integrated and operational methodology to screen and test aromas for OISE purposes. We demonstrated that odour quality and intensity is a key driving factor of OISE potency. Health authorities often recommend, besides a 
reduction in salt content of food, a lowering of fat and sugar content. It is likely that odour-induced taste enhancement could also be used to compensate the reduction of these tastants and the present methodology would be helpful to find candidate flavours (e.g. "butter" or "cream" odour) to enhance the fat or creamy perception (Bult, de Wijk, \& Hummel, 2007).

However, the present results were obtained with water solutions and sodium chloride. Sugar and fat are known to highly influence texture and mouthfeel of food products. Therefore, all sensory aspects of food products reduced in salt, sugar and fat need to be addressed, to reformulate food products that would be accepted by consumers.

\section{Acknowledgements}

This work was supported by INRA, Regional Council of Burgundy and Unilever R \& D Vlaardingen. Aromas were donated by Symrise and Givaudan (France).

\section{References}

Breslin, P. A. S., \& Beauchamp, G. K. (1997). Salt enhances flavor by suppressing bitterness. Nature, 387, 563.

Bult, J. H. F., de Wijk, R. A., \& Hummel, T. (2007). Investigations on multimodal sensory integration: Texture, taste, and ortho- and retronasal olfactory stimuli in concert. Neuroscience Letters, 411(1), 6-10.

Clark, C. C., \& Lawless, H. T. (1994). Limiting response alternatives in time-intensity scaling: An examination of the halo-dumping effect. Chemical Senses, 19, 583-594.

Cliff, M., \& Noble, A. C. (1990). Time-intensity evaluation of sweetness and fruitness and their interaction in a model solution. Journal of Food Science, 55, 450-454.

Djordjevic, J., Zatorre, R. J., \& Jones-Gotman, M. (2004a). Effects of perceived and imagined odours on taste detection. Chemical Senses, 29, 199-208.

Djordjevic, J., Zatorre, R. J., \& Jones-Gotman, M. (2004b). Odour-induced changes in taste perception. Experimental Brain Research, 159, 405-408.

Favier, J. C., Ireland-Ripert, J., Toque, C., \& Feinberg, M. (1995). Répertoire général des aliments - table de composition. Paris (FRA): Tec \& Doc.

Frank, R. A., \& Byram, J. (1988). Taste-smell interactions are tastant and odorant dependent. Chemical Senses, 13, 445-455.

Frank, R. A., Van der Klaauw, N. J., \& Schifferstein, H. N. J. (1993). Both perceptual and conceptual factors influence taste-odour and taste-taste interactions. Perception \& Psychophysics, 54, 343-354.

Girgis, S., Neal, B., Prescott, J., Prendergast, J., Dumbrell, S., Turner, C., et al. (2003). A one-quarter reduction in the salt content of bread can be made without detection. European Journal of Clinical Nutrition, 57, 616-620.

Hewson, L., Hollowood, T., Chandra, S., \& Hort, J. (2008). Taste-aroma interactions in a citrus flavoured model beverage system: Similarities and differences between acid and sugar type. Food Quality and Preference, 19, 323-334.

Keast, R. S. J., \& Breslin, P. A. S. (2002). An overview of binary taste-taste interactions. Food Quality and Preference, 14, 111-124.
Kuo, Y. L., Pangborn, R. M., \& Noble, A. C. (1993). Temporal patterns of nasal, oral, and retronasal perception of citral and vanillin and interaction of these odourants with selected tastants. International Journal of Food Science and Technology, 28, 127-137.

Labbe, D., Damevin, L., Vaccher, C., Morgenegg, C., \& Martin, N. (2006). Modulation of perceived taste by olfaction in familiar and unfamiliar beverages. Food Quality and Preference, 17, 582-589.

Linforth, R., Martin, F., Carey, M., Davidson, J., \& Taylor, A. J. (2002). Retronasal transport of aroma compounds. Journal of Agricultural and Food Chemistry, 50, 1111-1117.

Nguyen, D. H., Valentin, D., Ly, M. H., Chrea, C., \& Sauvageot, F. (2002). When does smell enhance taste? Effect of culture and odorant/tastant relationship. Paper presented at the European chemoreception research organisation conference. Erlangen, Germany, 23-27 July.

Pionnier, E., Nicklaus, S., Chabanet, C., Mioche, L., Taylor, A. J., Le Quéré, J. L., et al. (2004). Flavor perception of a model cheese: Relationships with oral and physico-chemical parameters. Food Quality and Preference, 15, $843-852$.

Prescott, J. (2004). Psychological process in flavour perception. In A. J. Taylor \& D. D. Robert (Eds.), Flavour perception (pp. 256-277). Blackwell publishing.

Prescott, J., Johnstone, V., \& Francis, J. (2004). Odor-taste interactions: Effects of attentional strategies during exposure. Chemical Senses, 29, 331-340.

Rolls, E. T., \& Baylis, L. L. (1994). Gustatory, olfactory, and visual convergence within the primate orbitofrontal cortex. Journal of Neuroscience, 14, 5437-5452.

Saint-Eve, A., Paci Kora, E., \& Martin, N. (2004). Impact of the olfactory quality and chemical complexity of the flavouring agent on the texture of low fat stirred yogurts assessed by three different sensory methodologies. Food Quality and Preference, 15, 655-668.

Sakai, N., Kobayakawa, T., Gotow, N., Saito, S., \& Imada, S. (2001). Enhancement of sweetness ratings of aspartame by a vanilla odour presented either by orthonasal or retronasal routes. Perceptual and Motor Skills, 92(3 Pt 2), 1002-1008.

Salles, C. (2006). Odour-taste interactions in flavour perception. In A. Voilley \& P. Etiévant (Eds.), Flavour in food (pp. 345-368). Woodhead Publishing Limited.

Schifferstein, H. (1997). Perceptual and imaginary mixtures in chemosensation. Journal of Experimental Psychology: Human Perception and Performance, 23, 278-288.

Schifferstein, H. N. J., \& Verlegh, P. W. J. (1996). The role of congruency and pleasantness in odor-induced taste-enhancement. Acta Psychologica, 94 $87-105$.

Small, D. M., \& Prescott, J. (2005). Odour/taste integration and the perception of flavor. Experimental Brain Research, 166, 345-357.

Stevenson, R. J., Prescott, J., \& Boakes, R. A. (1999). Confusing tastes and smells: How odours can influence the perception of sweet and sour tastes. Chemical Senses, $24,627-635$.

Stevenson, R. J., \& Tomiczek, C. (2007). Olfactory-induced synesthesias: A review and model. Psychological Bulletin, 133, 294-309.

Valentin, D., Chrea, C., \& Nguyen, D. H. (2006). Taste-odour interaction in sweet taste perception. In W. J. Spillane (Ed.), Optimising sweet taste in foods (pp. 66-84). Woodhead Publishing Limited.

Verhagen, J. V., \& Engelen, L. (2006). The neurocognitive bases of human multimodal food perception: Sensory integration. Neuroscience \& Biobehavioral Reviews, 30 , 613-650.

World Health Organisation (2007). Reducing salt intake in populations. Report of a WHO forum and technical meeting. Geneva, Switzerland: WHO Document Production Services. 